\title{
Comparison of acute cholangitis with or without common bile duct dilatation
}

\author{
MINORU TOMIZAWA ${ }^{1}$, FUMINOBU SHINOZAKI ${ }^{2}$, RUMIKO HASEGAWA $^{3}$, YOSHINORI SHIRAI ${ }^{3}$, \\ YASUFUMI MOTOYOSHI ${ }^{4}$, TAKAO SUGIYAMA ${ }^{5}$, SHIGENORI YAMAMOTO $^{6}$ and NAOKI ISHIGE ${ }^{7}$ \\ Departments of ${ }^{1}$ Gastroenterology, ${ }^{2}$ Radiology, ${ }^{3}$ Surgery, ${ }^{4}$ Neurology, ${ }^{5}$ Rheumatology, ${ }^{6}$ Pediatrics and \\ ${ }^{7}$ Neurosurgery, National Hospital Organization, Shimoshizu Hospital, Yotsukaido, Chiba 284-0003, Japan
}

Received October 15, 2015; Accepted February 17, 2017

DOI: $10.3892 / \mathrm{etm} .2017 .4401$

\begin{abstract}
To improve the management of patients with acute cholangitis, the present study compared laboratory test variables between acute cholangitis patients with or without common bile duct (CBD) dilatation [CBDdil(+) and CBDdil(-), respectively]. The medical records of patients diagnosed with acute cholangitis and subjected to endoscopic retrograde cholangiopancreatography between February 2008 and May 2015 were retrospectively analyzed. The present study consisted of 40 men (aged 69.4 \pm 8.8 years) and 37 women (aged $68.8 \pm 11.6$ years). It was observed that CBDdil(-) patients were slightly younger than CBDdil(+) patients $(\mathrm{P}=0.0976)$, and levels of $\mathrm{C}$-reactive protein $(\mathrm{CRP})$ were significantly higher in CBDdil(-) patients than in CBDdil(+) patients ( $\mathrm{P}=0.0392)$. In addition, logistic regression analysis indicated that CRP levels were associated with the presence of CBD dilatation $(\mathrm{P}=0.0392)$. These data indicate that patients with acute cholangitis without CBD dilatation tend to be younger and have higher levels of CRP. Thus, in acute cholangitis patients without CBD dilatation, diagnosis should be determined using clinical symptoms and laboratory data.
\end{abstract}

\section{Introduction}

Acute cholangitis is a bacterial inflammation that develops due to obstruction of the bile duct (1-3), typically by gallstones. A total of $76.6 \%$ of acute cholangitis cases are caused by gram-negative bacteria, including Escherichia coli and Klebsiella pneumoniae (4). Acute cholangitis may progress to sepsis, which in some cases is fatal, as the bacteria are able to translocate to the blood $(5,6)$. In the clinical management of acute cholangitis, biliary drainage is generally performed (7)

Correspondence to: Dr Minoru Tomizawa, Department of Gastroenterology, National Hospital Organization Shimoshizu Hospital, 934-5 Shikawatashi, Yotsukaido, Chiba 284-0003, Japan E-mail: nihminor-cib@umin.ac.jp

Key words: acute cholangitis, diameter, common bile duct by endoscopic retrograde cholangiopancreatography (ERCP), percutaneous transhepatic cholangiodrainage and endoscopic ultrasound-guided drainage (8). For prompt treatment of patients with acute cholangitis, accurate diagnosis is necessary.

Acute cholangitis is currently diagnosed based on evidence of bile duct obstruction and inflammation (9). Evidence of inflammation includes the presence of fever and elevations in white blood cell (WBC) count and C-reactive protein (CRP) levels, while bile duct obstruction is identified by elevated levels of liver enzymes, including total bilirubin (T-Bil), alkaline phosphatase (ALP), aspartate aminotransferase (AST), alanine aminotransferase (ALT) and $\gamma$-glutamyl transpeptidase $(\gamma$-GTP).

Diagnosis of acute cholangitis is typically confirmed by diagnostic imaging (9), including abdominal ultrasound (US), computed tomography (CT) and magnetic resonance cholangiopancreatography (MRCP). Abdominal US and CT are able to identify bile duct obstruction, dilatation of the common bile duct (CBD) and gallstones (10). For patients with contraindications to MRCP, the 3D structure of the CBD may be visualized with CT (11). However, these diagnostic imaging techniques primarily evaluate CBD dilatation, thus it is difficult to use imaging techniques to diagnose acute cholangitis in patients without $\mathrm{CBD}$ dilatation.

Therefore, the present study retrospectively compared the laboratory test variables and ages of acute cholangitis patients with or without CBD dilatation, with the aim of applying these results to improve patient management.

\section{Patients and methods}

Diagnosis and management of acute cholangitis. Patients at the National Hospital Organization Shimoshizu Hospital were diagnosed with acute cholangitis using the clinical Charcot's triad criteria (Table I) (8) or the criteria for 'suspected' or 'definite' acute cholangitis according to the Tokyo guidelines (TG13) (9). Briefly, patients were diagnosed with acute cholangitis when they had the following Charcot's triad: Fever, jaundice and abdominal pain. However, not all patients with acute cholangitis meet the Charcot's triad criteria (12). Therefore, when patients did not meet the Charcot's triad criteria but were suspected of having acute 
Table I. Diagnostic criteria for acute cholangitis based on Charcot's triad and the TG13.

Charcot's triad

TG13

Fever, jaundice, abdominal pain A. Systemic inflammation

Fever and/or shaking chills, laboratory data, evidence of inflammatory response (WBC count andCRP levels)

B. Cholestasis

Jaundice, laboratory data, abnormal liver function tests (ALP, AST, ALT and $\gamma$-GTP levels)

C. Imaging

Biliary dilatation, evidence of disease etiology (including structure and stones)

Patients were diagnosed with acute cholangitis if they met the Charcot's triad criteria (8). If patients did not meet the Charcot's triad criteria, they were diagnosed with acute cholangitis if they met the criteria for suspected (one item in A + one item in either B or C) or definite (one item in each of A, B and C) acute cholangitis according to the TG13 (9). Imaging was performed with abdominal ultrasonography, computed tomography and magnetic resonance cholangiopancreatography. TG13, Tokyo guidelines 2013 edition; WBC, white blood cell; CRP, C-reactive protein; ALP, alkaline phosphatase; AST, aspartate aminotransferase; ALT, alanine aminotransferase; $\gamma$-GTP, $\gamma$-glutamyl transpeptidase.

cholangitis, they were evaluated according to the Tokyo guidelines (9). A total of 17 patients were diagnosed with acute cholangitis according to the Charcot's triad, while 60 patients were diagnosed based on the TG13. Diagnostic imaging, including abdominal US, CT and MRCP, was performed to investigate the etiology of acute cholangitis. Following diagnosis of acute cholangitis, patients were treated with an intravenous infusion of cephazolin $(3 \mathrm{~g} /$ day; Nipro Corporation, Osaka, Japan) while fasting, as described previously (13). The severity of acute cholangitis was assessed according to the Tokyo guidelines, as revised by Kiriyama et al (9). All enrolled patients received ERCP for drainage and symptom management. No patients exhibited shock, acute lung failure, acute renal failure, hepatic failure or disseminated intravascular coagulation. Patients did not undergo ERCP when they recovered from acute cholangitis or did not wish to undergo ERCP.

Inclusion and exclusion criteria. Patients were enrolled in the current study if they met the following inclusion criteria: i) Diagnosis with acute cholangitis according to the aforementioned criteria, ii) treatment with ERCP, and iii) hospitalization in the National Hospital Organization Shimoshizu Hospital between February 2008 and May 2015. Patients were excluded from the current study when they met the following exclusion criteria: i) Diagnosis with acute cholangitis but not subjected to ERCP, and ii) subjected to ERCP for reasons other than acute cholangitis. A total of 40 men (aged $69.4 \pm 8.8$ years) and 37 women (aged $68.8 \pm 11.6$ years) were enrolled in the current study. The cause of acute cholangitis in all but one patient was a CBD stone or sludge. The remaining patient had cancer of the CBD and a CBD stone. The current study was approved by the Ethics Committee of the National Hospital Organization Shimoshizu Hospital (Yotsukaido, Japan). Written informed consent was obtained from patients prior to MRCP, ERCP and CT with or without contrast enhancement. Informed consent was obtained from patients prior to abdominal US, though written informed consent was waived as abdominal US is considered to be safe and non-invasive. The current study was not considered a clinical trial as procedures were performed as part of routine clinical practice. Therefore, written informed consent for inclusion in the study was waived. All patient records were anonymized prior to retrospective analysis.

ERCP. Patients underwent moderate-level sedation with an intramuscular injection of $20 \mathrm{mg}$ hydroxyzine (Pfizer, Inc., New York, NY, USA) and intravenous injection of $5 \mathrm{mg}$ diazepam (Takeda, Osaka, Japan), and were under continuous cardiopulmonary monitoring. All ERCP procedures were performed by clinical endoscopists using an Evis Lucera duodenovideoscope JF-260V (Olympus Corporation, Tokyo, Japan). Cannulation of the bile duct was performed in all patients with a standard type cannula (PR-V216Q; Olympus Corporation). The cannula was used to detect CBD stones by fluoroscopy using a contrast medium (sodium meglumine diatrizoate; Bayer AG, Leverkusen, Germany). The cannula was then changed to a sphincterotome (KD-V411M-0720; Olympus Corporation) with a guidewire and endoscopic papillotomy (EPT) was performed. The CBD stones and/or sludge were removed with a balloon catheter (B-V232P-A; Olympus Corporation). For large or hard CBD stones, lithotripsy was initially performed with a lithotripter (BML-V437QR-30; Olympus Corporation), and the fractured $\mathrm{CBD}$ stones were removed with the lithotripter or balloon catheter. A nasobiliary catheter (NB-ST-CHBIN-72-3000-SH3-WP; Hanaco Medical Co., Ltd., Saitama, Japan) was inserted for drainage if necessary. Bile culture was not performed with ERCP. Following ERCP, patients were monitored for ERCP-related complications, including bleeding, perforation and/or pancreatitis. No such complications were observed.

Grouping of patients. The CBD diameter was measured using images from the ERCP procedure. Based on CBD measurements, enrolled patients were divided into the following two groups: A CBDdil(-) group for patients with a CBD diameter of $\leq 7.0 \mathrm{~mm}$ and a CBDdil(+) group for patients with a CBD diameter of $>7.0 \mathrm{~mm}$. Previous studies indicate that the upper 
Table II. Comparison of laboratory test variables in acute cholangitis patients with or without CBD dilatation.

\begin{tabular}{|c|c|c|c|c|}
\hline \multirow[b]{2}{*}{ Variable } & \multirow[b]{2}{*}{ Normal range } & \multicolumn{2}{|c|}{ CBD } & \multirow[b]{2}{*}{ P-value } \\
\hline & & $(-)$ & $(+)$ & \\
\hline No. of patients, male/female (ratio \%) & & $10 / 13(43.5 / 56.5)$ & $30 / 24(55.6 / 44.4)$ & 0.3314 \\
\hline Age, years & & $66.2 \pm 10.3$ & $70.4 \pm 10.0$ & 0.0976 \\
\hline CBD diameter, $\mathrm{mm}$ & $\leq 7.0$ & $4.1 \pm 1.5$ & $11.2 \pm 2.9$ & $<0.0001^{\mathrm{b}}$ \\
\hline $\mathrm{CBD}$ diameter range, $\mathrm{mm}$ & & $3.0-6.8$ & $7.2-18.0$ & \\
\hline $\mathrm{WBC}, \times 10^{3} / \mu 1$ & $3.4-8.5$ & $8.6 \pm 4.2$ & $8.7 \pm 5.2$ & 0.9187 \\
\hline $\mathrm{CRP}, \mathrm{mg} / \mathrm{dl}$ & $0.0-0.3$ & $7.1 \pm 8.8$ & $3.7 \pm 5.0$ & $0.0392^{\mathrm{a}}$ \\
\hline $\mathrm{T}-\mathrm{Bil}, \mathrm{mg} / \mathrm{dl}$ & $0.3-1.2$ & $21.4 \pm 16.7$ & $23.8 \pm 34.1$ & 0.7529 \\
\hline ALP, IU/1 & $115-339$ & $588 \pm 443$ & $630 \pm 414$ & 0.7010 \\
\hline AST, IU/1 & $13-33$ & $181 \pm 220$ & $145 \pm 218$ & 0.5135 \\
\hline ALT, IU/1 & $6-27$ & $220 \pm 281$ & $167 \pm 199$ & 0.3684 \\
\hline$\gamma$-GTP, IU/1 & $10-47$ & $286 \pm 240$ & $404 \pm 370$ & 0.1839 \\
\hline
\end{tabular}

Enrolled patients were divided into two groups: CBDdil(-) group for patients with a CBD diameter $\leq 7.0 \mathrm{~mm}$ and a CBDdil(+) group for patients with a CBD diameter $>7.0 \mathrm{~mm}$. Age and laboratory test variables of CBDdil(-) and CBDdil(+) patients were compared. CRP levels are presented as $\mathrm{mg} / \mathrm{dl}$, as described previously (8). Data are presented as the mean \pm standard deviation. ${ }^{\mathrm{a} P}<0.05,{ }^{\mathrm{b}} \mathrm{P}<0.0001$. CBD, common bile duct; (-), CBD dilatation absent; (+), CBD dilatation present; WBC, white blood cell count; CRP, C-reactive protein; T-Bil, total bilirubin; ALP, alkaline phosphatase; AST, aspartate aminotransferase; ALT, alanine aminotransferase; $\gamma$-GTP, $\gamma$-glutamyl transpeptidase; IU, international unit.

Table III. Pulse sequences used in the present study.

\begin{tabular}{lccc}
\hline Parameter & T1-weighted image & T2-weighted image & DWI \\
\hline Echo & GRE & Single-shot SE & EPI SE \\
TR, msec & Shortest & 1,000 & 5,000 \\
TE, msec & Shortest & 90 & 70 \\
Flip angle, & 75 & 90 & 90 \\
NSA & 1 & 1 & 4 \\
Slice thickness, mm & 8 & 8 & 5 \\
Slice gap & 1 & 1 & 0 \\
Fat saturation & no & no & SPAIR \\
Phase encoding direction & Posterior-anterior & Posterior-anterior & Posterior-anterior
\end{tabular}

TR, repetition time; TE, echo time; DWI, diffusion-weighted imaging; GRE, gradient echo; SE, spin echo; EPI, echo planar imaging; SPAIR, spectral attenuated inversion recovery; NSA, number of signal average.

limit of the CBD diameter in acute cholangitis ranges from 6.0 to $8.0 \mathrm{~mm}(14,15)$. In the current study, $7.0 \mathrm{~mm}$ was selected as the upper CBD diameter limit as this value is directly between 6.0 and $8.0 \mathrm{~mm}$. The CBDdil(-) group was comprised of 10 men and 13 women and the CBDdil(+) group consisted of 30 men and 24 women (Table II). The gender ratio did not differ significantly between the two groups $(\mathrm{P}=0.3314)$.

Laboratory test variables. Laboratory test variables evaluated in the current study included markers of inflammation and bile duct obstruction. The extent of inflammation was determined from the WBC count and CRP levels of patients. The extent of bile duct obstruction was determined by measuring levels of
T-Bil, ALP, AST, ALT and $\gamma$-GTP in patients. Abdominal US, MRCP, ERCP and CT diagnostic images were used to evaluate dilatation of the bile ducts. CRP levels were expressed in units of $\mathrm{mg} / \mathrm{dl}(9,16)$.

Imaging diagnostics. Abdominal US was performed with a SSA-700A ultrasound (Toshiba Medical Systems Corporation, Otawara, Japan). The probes used were a 3.75-MHz curved-array probe (PVT-375BT) or a 3-MHz sector-array probe (PST-308T; both from Toshiba Medical Systems Corporation). MRCP was performed with a 1.5 Tesla scanner using 3.2.2 Achieva software (Philips Medical Systems B.V., Eindhoven, The Netherlands). The MRCP pulse sequences are presented in Table III. CT was performed with a 16-detector-row CT scanner (Siemens AG, 
Munich, Germany). CT images were acquired at $130 \mathrm{kV}$ and $200 \mathrm{~mA}$ with a rotation time of $0.6 \mathrm{sec}$ and slice pitch of 0.8 .

Statistical analysis. Data are presented as the mean \pm standard deviation. A $\chi^{2}$ test was performed to analyze associations between men and women, the presence of a gallbladder stone and a history of cholecystectomy in the CBDdil(-) and CBDdil(+) groups. One-way analysis of variance followed by a Tukey's post hoc test was used to compare the age of patients and laboratory test variables between the CBDdil(-) and CBDdil(+) groups. Logistic regression analysis was performed to identify factors affecting dilatation of the CBD. All statistical analyses were performed with JPM 10.0.2 software (SAS Institute, Inc., Cary, NC, USA) and $\mathrm{P}<0.05$ was considered to indicate a statistically significant difference.

\section{Results}

Typical cases of CBDdil(-) and CBDdil( + ). In one representative patient with CBDdil(+), a CBD stone was identified by CT, abdominal US, MRCP and ERCP (Fig. 1A-D). EPT was performed and the stone was removed (Fig. 1Eand F). In one representative patient with CBDdil(-), sludge was identified by abdominal US (Fig. 2A), and MRCP and ERCP revealed that there was no dilatation of the CBD (Fig. 2B and C). Secretions of bile containing sludge from the Ampulla of Vater were also observed (Fig. 2D). CBD stones were identified in two CBDdil(-) patients. Sludge was not detected in any CBDdil(+) patients with abdominal US, CT, or MRCP.

Laboratory test variables. Potential differences between the laboratory test variables and ages of CBDdil(-) and CBDdil(+) patients were evaluated (Table II). The gender ratio did not differ significantly between the CBDdil(-) and CBDdil(+) groups. Patients in the CBDdil(-) group were younger than those in the CBDdil(+) group, although this difference was not significant $(66.2 \pm 10.3$ vs. $70.4 \pm 10.0 ; \mathrm{P}=0.0976)$. CBD diameter was significantly larger in $\operatorname{CBDdil}(+)$ patients than in CBDdil(-) patients $(\mathrm{P}<0.0001)$. CBDdil(-) patients had significantly higher levels of CRP than CBDdil(+) patients (7.1 \pm 8.8 vs. $3.7 \pm 5.0 ; P=0.0392)$. WBC count and liver enzyme levels did not significantly differ between the CBDdil(-) and CBDdil(+) groups. These data are consistent with previous results indicating that the CBD diameter is generally larger in older patients (14). In addition, the present results suggest that levels of CRP may be associated with CBD dilatation.

To determine whether CBD dilatation was associated with the presence of a gallbladder stone or history of cholecystectomy, a $\chi^{2}$ test was performed (Table IV). It was determined that the presence of a gallbladder stone was not correlated with $\mathrm{CBD}$ dilatation $(\mathrm{P}=0.7774)$. There was also no correlation between history of cholecystectomy and CBD dilatation $(\mathrm{P}=0.1389)$. These results suggest that the presence of a gallbladder stone or a history of cholecystectomy has no significant effect on CBD dilatation.

Logistic regression analysis was performed to determine whether patient laboratory test variables or age were associated with the presence of CBD dilatation (Table V). Age and $\mathrm{CRP}$ levels were associated with the presence of CBD dilatation $(\mathrm{P}=0.0193$ and $\mathrm{P}=0.0075$, respectively). $\mathrm{WBC}$ count and

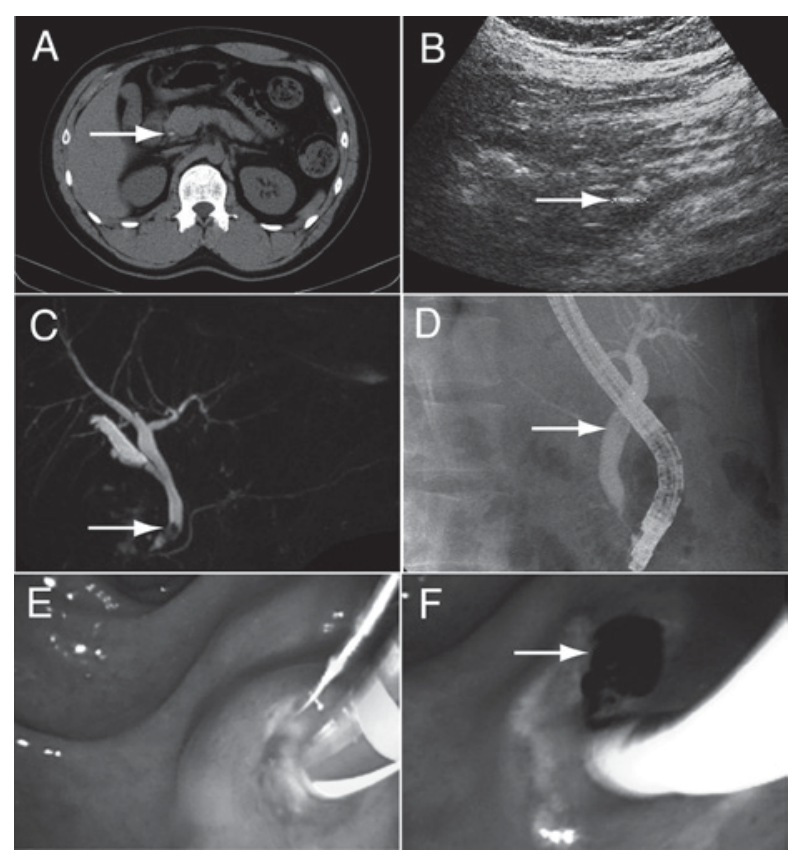

Figure 1. A representative case of an acute cholangitis patient with CBD dilatation. A 41-year-old man was admitted to the National Hospital Organization Shimoshizu Hospital presented with right upper quadrant pain. (A) Computed tomography and (B) abdominal ultrasonography revealed a high-intensity signal and strong echo in the CBD, respectively (white arrows). (C) Magnetic resonance cholangiopancreatography and (D) endoscopic retrograde cholangiopancreatography identified a defect in the CBD (white arrows). (E) Endoscopic sphincterotomy was performed and (F) a gallstone (white arrow) was removed CBD, common bile duct.
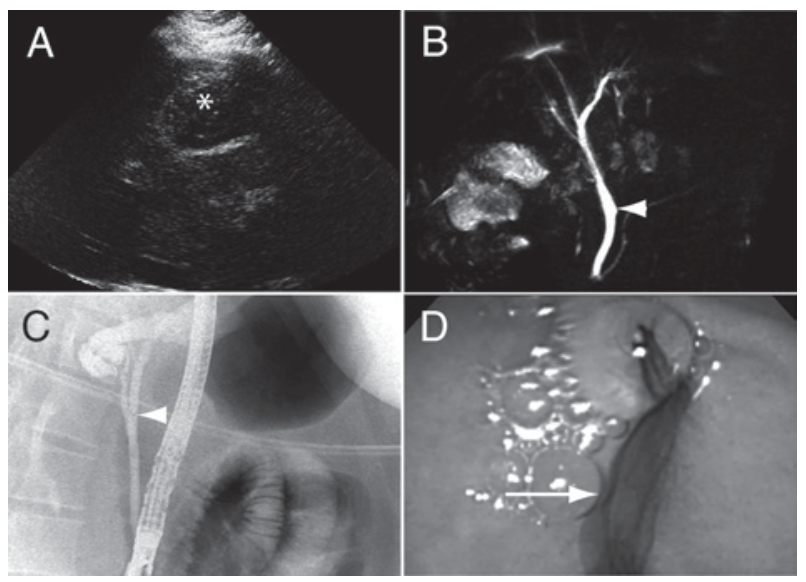

Figure 2. Acute cholangitis patient without CBD dilatation. A 42-year-old man was hospitalized with myotonic dystrophy. The patient presented with abdominal pain, fever and jaundice. (A) Abdominal ultrasonography identified sludge (asterisk) in the gallbladder. (B) Magnetic resonance cholangiopancreatography and (C) ERCP demonstrated that the CBD (arrowhead) was not dilated. (D) ERCP identified secretions of bile (arrow) and sludge from the Ampulla of Vater. No CBD stones were observed by ERCP. CBD, common bile duct; ERCP, endoscopic retrograde cholangiopancreatography.

liver enzyme levels were not associated with the presence of CBD dilatation.

\section{Discussion}

CBD dilatation is caused by an impairment of bile flow $(17,18)$, which in turn impacts on laboratory test variables. However, 
Table IV. Association of CBD dilatation with the presence of a gallbladder stone or history of cholecystectomy.

Common bile duct dilatation

\begin{tabular}{lccr}
\cline { 2 - 3 } Variable & $(-)$ & $(+)$ & P-value \\
\hline GB stone, -/+ (ratio \%) & $9 / 14(39.1 / 60.9)$ & $23 / 31(42.6 / 57.4)$ & 0.7774 \\
No. of patients with cholecystectomy, -/+ (ratio \%) & $23 / 0(100.0 / 0.0)$ & $51 / 3(94.4 / 5.6)$ & 0.1389
\end{tabular}

A $\chi^{2}$ test was performed to determine whether CBD dilatation was associated with the presence of a gallbladder stone or history of cholecystectomy. (-), bile duct dilatation, gallbladder stone or history of cholecystectomy absent; (+), bile duct dilatation, gallbladder stone or history of cholecystectomy present. CBD, common bile duct; GB, gallbladder.

Table V. Logistic regression analysis of laboratory test variables.

\begin{tabular}{lccl}
\hline Variable & OR & $95 \%$ CI & P-value \\
\hline Age & 0.9423 & $0.8786-1.0024$ & $0.0193^{\mathrm{a}}$ \\
WBC & 0.9998 & $0.9996-1.0000$ & 0.1648 \\
CRP & 1.1952 & $1.0589-1.3990$ & $0.0075^{\mathrm{b}}$ \\
T-Bil & 0.8398 & $0.5607-1.0889$ & 0.5209 \\
ALP & 1.0003 & $0.9984-1.0021$ & 0.7491 \\
AST & 1.0021 & $0.9976-1.0064$ & 0.2201 \\
ALT & 1.0002 & $0.9945-1.0055$ & 0.8946 \\
$\gamma$-GTP & 0.9983 & $0.9951-1.0008$ & 0.1318 \\
\hline
\end{tabular}

Logistic regression analysis was performed to determine whether patient laboratory test variables or age were associated with the presence of CBD dilatation. ${ }^{a} \mathrm{P}<0.05$ and ${ }^{\mathrm{b}} \mathrm{P}<0.01$. OR, odds ratio; $\mathrm{CI}$, confidence interval; WBC, white blood cell count; CRP, C-reactive protein; T-Bil, total bilirubin; ALP, alkaline phosphatase; AST, aspartate aminotransferase; ALT, alanine aminotransferase; $\gamma$-GTP, $\gamma$-glutamyl transpeptidase.

some patients with acute cholangitis present with CBD inflammation without dilatation. Hong et al (19) demonstrated that acute cholangitis without CBD dilatation is caused by CBD stones and sludge, with the latter also being implicated in the development of acute cholangitis (20). Interestingly, in one CBDdil(-) patient included in the present study, secretions of bile and sludge from the Ampulla of Vater were observed, indicating that sludge may be present during CBD without CBD dilatation. Collectively, these results suggest that gallbladder sludge may be a cause of acute cholangitis without $\mathrm{CBD}$ dilatation. By contrast, the present study observed that levels of T-Bil, ALP, AST, ALT and $\gamma$-GTP did not differ significantly between CBDdil(-) and CBDdil(+) patients. This discrepancy with previous results may be due to the relatively small sample size of the current study.

CRP is an acute-phase protein and levels of CRP increase in the blood during inflammation (21). In the present study, CRP levels were significantly higher in CBDdil(-) patients than in CBDdil(+) patients. Previous studies have demonstrated that increased levels of CRP, WBCs and T-Bil are associated with severe acute cholangitis and poor patient prognosis $(22,23)$. In the current study, WBC count and T-Bil levels did not differ significantly between the CBDdil(-) and CBDdil(+) groups, suggesting that the severity of acute cholangitis was similar between the groups. Thus, there may be alternative explanations for the observed elevation in CRP levels in CBDdil(-) patients other than the severity of acute cholangitis. For instance, as CRP is primarily synthesized in the liver in response to interleukin-6 (24), the hepatic production rate of CRP may differ between CBDdil(-) and CBDdil(+) patients. However, the underlying mechanisms responsible for the higher levels of CRP observed in CBDdil(-) patients remain unknown.

Although CBD dilatation aids in the diagnosis of acute cholangitis (9), some patients with acute cholangitis do not exhibit CBD dilatation. For patients without $\mathrm{CBD}$ dilatation, imaging diagnostic techniques may allow accurate diagnosis of acute cholangitis. Other indications of CBD inflammation include an increase in magnetic resonance imaging (MRI) signal intensity in the region surrounding the CBD on T2-weighted images (25), or the presence of an abscess near the CBD on CT and MRI images $(10,25)$. As well as imaging diagnostics, laboratory test variables may aid in the diagnosis of acute cholangitis (26). The present study identified an association between CRP levels and the presence of CBD dilatation; thus laboratory test variables may be potential diagnostic factors for the accurate diagnosis of acute cholangitis. Evaluation of the CBD diameter itself may also be key in the diagnosis of acute cholangitis (27).

One limitation of the current study was the small number of patients enrolled. For example, levels of $\gamma$-GTP tended to be higher in CBDdil(+) patients than in CBDdil(-) patients, indicating that bile flow may have been disturbed. However this difference was not significant $(\mathrm{P}=0.1839)$, possibly due to the small sample size of the current study (17).

In conclusion, acute cholangitis patients without CBD dilatation were slightly younger and exhibited significantly higher levels of CRP than patients with CBD dilatation. These data indicate that diagnosis of acute cholangitis in patients without CBD dilatation should be based on a combination of clinical symptoms and laboratory data.

\section{References}

1. Mosler P: Management of acute cholangitis. Gastroenterol Hepatol (N Y) 7: 121-123, 2011.

2. Sahu MK, Chacko A, Dutta AK and Prakash JA: Microbial profile and antibiotic sensitivity pattern in acute bacterial cholangitis. Indian J Gastroenterol 30: 204-208, 2011.

3. Kim SW, Shin HC, Kim HC, Hong MJ and Kim IY: Diagnostic performance of multidetector $\mathrm{CT}$ for acute cholangitis: Evaluation of a CT scoring method. Br J Radiol 85: 770-777, 2012. 
4. Karpel E, Madej A, Bułdak Ł, Duława-Bułdak A Nowakowska-Duława E, Łabuzek K, Haberka M, Stojko R and Okopień B: Bile bacterial flora and its in vitro resistance pattern in patients with acute cholangitis resulting from choledocholithiasis. Scand J Gastroenterol 46: 925-930, 2011.

5. Qureshi WA: Approach to the patient who has suspected acute bacterial cholangitis. Gastroenterol Clin North Am 35: 409-423, 2006.

6. Yoneyama K, Saito H, Kurihara T, Kogo M, Kitamura K, Iwata $\mathrm{T}$, Imawari $\mathrm{M}$ and Kiuchi $\mathrm{Y}$ : Factors involved in resistance to early treatment of acute cholangitis patients. Hepatogastroenterology 59: 1722-1726, 2012.

7. Shinya S, Sasaki T, Yamashita Y, Kato D, Yamashita K, Nakashima R, Yamauchi Y and Noritomi T: Procalcitonin as a useful biomarker for determining the need to perform emergency biliary drainage in cases of acute cholangitis. J Hepatobiliary Pancreat Sci 21: 777-785, 2014.

8. Mosler P: Diagnosis and management of acute cholangitis. Curr Gastroenterol Rep 13: 166-172, 2011.

9. Kiriyama S, Takada T, Strasberg SM, Solomkin JS, Mayumi T, Pitt HA, Gouma DJ, Garden OJ, Büchler MW, Yokoe M, et al: New diagnostic criteria and severity assessment of acute cholangitis in revised Tokyo Guidelines. J Hepatobiliary Pancreat Sci 19: 548-556, 2012.

10. Patel NB, Oto A and Thomas S: Multidetector CT of emergent biliary pathologic conditions. Radiographics 33: 1867-1888, 2013.

11. Zhang ZY, Wang D, Ni JM, Yu XR, Zhang L, Wu WJ, Gong L and $\mathrm{Hu} \mathrm{MH}$ : Comparison of three-dimensional negative-contrast CT cholangiopancreatography with three-dimensional MR cholangiopancreatography for the diagnosis of obstructive biliary diseases. Eur J Radiol 81: 830-837, 2012.

12. Rahman SH, Larvin M, McMahon MJ and Thompson D: Clinical presentation and delayed treatment of cholangitis in older people. Dig Dis Sci 50: 2207-2210, 2005.

13. Miura F, Takada T, Strasberg SM, Solomkin JS, Pitt HA, Gouma DJ, Garden OJ, Büchler MW, Yoshida M, Mayumi T, et al: TG13 flowchart for the management of acute cholangitis and cholecystitis. J Hepatobiliary Pancreat Sci 20 : 47-54, 2013.

14. Lal N, Mehra S and Lal V: Ultrasonographic measurement of normal common bile duct diameter and its correlation with age, sex and anthropometry. J Clin Diagn Res 8: AC01-AC04, 2014 .
15. Park SM, Kim WS, Bae IH, Kim JH, Ryu DH, Jang LC and Choi JW: Common bile duct dilatation after cholecystectomy: A one-year prospective study. J Korean Surg Soc 83: 97-101, 2012.

16. Gorska-Ciebiada M, Saryusz-Wolska M, Borkowska A, Ciebiada $\mathrm{M}$ and Loba J: C-reactive protein, advanced Glycation end products, and their receptor in type 2 diabetic, elderly patients with mild cognitive impairment. Front Aging Neurosci 7: 209, 2015.

17. Tomizawa M, Shinozaki F, Motoyoshi Y, Sugiyama T, Yamamoto S and Sueishi M: Association between juxtapapillary diverticulum and acute cholangitis determined using laboratory data. Clin Exp Gastroenterol 7: 447-451, 2014.

18. Kim CW, Chang JH, Kim JH, Kim TH, Lee IS and Han SW: Size and type of periampullary duodenal diverticula are associated with bile duct diameter and recurrence of bile duct stones. J Gastroenterol Hepatol 28: 893-898, 2013.

19. Hong MJ, Kim SW, Kim HC and Yang DM: Comparison of the clinical characteristics and imaging findings of acute cholangitis with and without biliary dilatation. Br J Radiol 85: e1219-e1225, 2012.

20. Lee YS, Kang BK, Hwang IK, Kim J and Hwang JH: Long-term outcomes of symptomatic gallbladder sludge. J Clin Gastroenterol 49: 594-598, 2015.

21. Markanday A: Acute phase reactants in infections: Evidence-based review and a guide for clinicians. Open Forum Infect Dis 2: ofv098, 2015.

22. Qin YS, Li QY, Yang FC and Zheng SS: Risk factors and incidence of acute pyogenic cholangitis. Hepatobiliary Pancreat Dis Int 11: 650-654, 2012.

23. Lee CC, Chang IJ, Lai YC, Chen SY and Chen SC: Epidemiology and prognostic determinants of patients with bacteremic cholecystitis or cholangitis. Am J Gastroenterol 102: 563-569, 2007.

24. Ansar W and Ghosh S: C-reactive protein and the biology of disease. Immunol Res 56: 131-142, 2013.

25. Eun HW, Kim JH, Hong SS and Kim YJ: Assessment of acute cholangitis by MR imaging. Eur J Radiol 81: 2476-2480, 2012.

26. Tomizawa M, Shinozaki F, Hasegawa R, Togawa A, Shirai Y, Ichiki N, Motoyoshi Y, Sugiyama T, Yamamoto S and Sueishi M: An association of elevated levels of alkaline phosphatase and gamma-glutamyl transpeptidase with acute cholangitis. Hepatogastroenterology 61: 291-295, 2014.

27. Horrow MM: Ultrasound of the extrahepatic bile duct: Issues of size. Ultrasound Q 26: 67-74, 2010. 